Questions vives

\section{Questions Vives}

Recherches en éducation

$N^{\circ} 25$ | 2016

L'activité des enseignants face au décrochage scolaire : Quelles difficultés et quelles mises en œuvre professionnelles?

\title{
La diversité des approches comme levier pour favoriser l'accrochage. Étude d'un dispositif pour élèves en rupture
}

The diversity of approaches as lever to enhance school engagement. Study of a program for students at risk

Chantal Tièche Christinat, Valérie Angelucci et Léonie Liechti

\section{(2) OpenEdition \\ Journals}

Édition électronique

URL : http://journals.openedition.org/questionsvives/1877

DOI : $10.4000 /$ questionsvives. 1877

ISSN : $1775-433 \mathrm{X}$

Éditeur

Université Aix-Marseille (AMU)

Édition imprimée

Date de publication : 7 juillet 2016

ISBN : 978-2-912643-49-0

ISSN : $1635-4079$

\section{Référence électronique}

Chantal Tièche Christinat, Valérie Angelucci et Léonie Liechti, «La diversité des approches comme levier pour favoriser l'accrochage. Étude d'un dispositif pour élèves en rupture », Questions Vives [En ligne], № 25 | 2016, mis en ligne le 05 septembre 2016, consulté le 02 mai 2019. URL : http:// journals.openedition.org/questionsvives/1877; DOI : 10.4000/questionsvives.1877

Ce document a été généré automatiquement le 2 mai 2019.

\section{cc) (†)}

Questions Vives est mis à disposition selon les termes de la licence Creative Commons Attribution -

Pas d'Utilisation Commerciale - Pas de Modification 4.0 International. 


\title{
La diversité des approches comme levier pour favoriser l'accrochage. Étude d'un dispositif pour élèves en rupture
}

\author{
The diversity of approaches as lever to enhance school engagement. Study of a \\ program for students at risk
}

Chantal Tièche Christinat, Valérie Angelucci et Léonie Liechti

\section{Introduction}

1 Les constats d'échec mettant en cause les objectifs des politiques scolaires et leur évocation se couplent fréquemment avec des considérations portant sur les phénomènes $\mathrm{du}$ décrochage scolaire et ses conséquences politiques économiques et sociales. Manifestation visible de l'échec scolaire ou même épiphénomène incontrôlé et assorti à celui-ci (Bernard, 2011), le décrochage scolaire constitue actuellement une préoccupation politique et économique importante, présentée dans les discours sur l'école comme le mal à combattre. Ce faisant, la problématique du décrochage pose une nouvelle fois la question fondamentale de l'inégalité devant la réussite scolaire et illustre à sa manière l'échec du système scolaire et des politiques publiques mises en place pour pallier les inégalités constatées (Broccolichi \& Ben-Ayed, 1999; Dubet, 2005). Démentant la promesse de l'égalité des chances, il met en lumière non seulement les lacunes de l'école et les échecs des maîtres (Dubet, 2014) mais impacte, comme le soulignent les coordinateurs de ce numéro, la forme scolaire et les habitudes du métier d'enseignant. Considéré en effet comme un problème majeur par les institutions éducatives qui ont pour injonction d'en diminuer la prévalence (Blaya, 2013), le décrochage scolaire interpelle les chercheurs en sciences de l'éducation non seulement en raison de la complexité du phénomène et des processus dynamiques qui le constituent (Tièche 
Christinat \& Gilles, 2013), mais aussi au vu de la fragilité des réponses apportées et des alliances éducatives construites pour favoriser la coordination d'actions éducatives et pédagogiques (Baeriswyl, Savoy, \& Tièche Christinat, 2013; Colombo, 2010; Flavier \& Moussay, 2014).

\subsection{Les déterminants du décrochage}

2 Si l'échec scolaire peut se définir par la qualité de rapport entre les facteurs sociaux et les facteurs structurels et organisationnels propres à l'institution scolaire (CRESAS, 1981), les travaux de recherche menés tant sur l'échec que sur le décrochage scolaire soulignent également le poids des facteurs pédagogiques, mettant en évidence des logiques et des pratiques d'acteurs susceptibles de créer des différences (Bonnéry, 2007) et de développer des mécanismes de démobilisation des élèves (Broccolichi \& Ben-Ayed, 1999). La fabrication du décrochage scolaire ou son enclenchement résulte ainsi d'une convergence de déterminants intrinsèques ou extrinsèques au système scolaire (Blaya, 2010 ; Fortin, Marcotte, Royer, \& Potvin, 2005a, 2005b ; Gilles, Tièche Christinat, \& Delévaux, 2012 ; Potvin, Fortin, \& Lessard, 2005). Les déterminants extrinsèques comprennent les facteurs sociaux recensés comme producteurs de la réussite ou de l'échec scolaire, en particulier l'environnement familial, culturel et social ainsi que les facteurs personnels relevant du rapport à soi et aux autres. Les déterminants intrinsèques, quant à eux, englobent les facteurs organisationnels et structurels liés à la classe, sa composition, sa situation et son environnement. Ils comprennent en sus les facteurs pédagogiques et relationnels liés aux pratiques et postures pédagogiques qui se manifestent au sein de l'école et de la classe.

3 Au vu de son caractère multidéterminé, complexe et dynamique, le décrochage scolaire se décline sous différentes formes et profils de décrocheurs, mettant à mal des réponses scolaires uniformes ou indifférenciées (Barrère, 2013). Pour faire face à une problématique scolaire et sociale considérée comme préoccupante et intensifier la lutte contre l'échec scolaire, une multiplicité de programmes et de dispositifs est proposée à divers moments du parcours scolaire aux enseignants, aux élèves et à leurs parents.

\subsection{Les dispositifs}

4 Construit à l'aulne des expériences précédentes inscrites dans la prévention de l'échec scolaire, ces nouvelles formes d'organisation scolaire affichent une variété et une hétérogénéité qui semblent pouvoir relever, comme le souligne Barrère (2013), tous les grands défis actuels de l'école. La diversité des publics et le nombre croissant d'élèves en difficulté ont convié les professionnels à diversifier les prises en charge et à favoriser l'invention de systèmes très souples permettant une diversification des modes de scolarité (Guigue, 2013). Chaque dispositif se distingue des autres en fonction des lieux de son implantation, des missions et des rôles dont ils sont porteurs ainsi que des facteurs sur lesquels les professionnels souhaitent agir.

5 L'examen de quelques programmes d'accrochage et des pratiques exercées en leur sein (Guigue, 2013a ; Hugon, \& Toubert-Duffort, 2011 ; Gilles, Potvin, \& Tièche Christinat, 2012) signale une prise en compte de facteurs relevant autant du système intrinsèque à l'école que de facteurs extrinsèques à celle-ci, tels que les caractéristiques sociales, familiales, éducatives ou personnelles de l'élève en situation de décrochage pointées par Fortin et ses collègues (2006). Les orientations adoptées par les dispositifs, qui semblent toutefois 
privilégier tantôt une volonté de restauration du pédagogique, tantôt une intention réparatrice ou de soin prodigué à l'élève (Tièche Christinat, Baeriswyl, Delévaux, Savoy, \& Cassagne, 2012), conduisent parfois à un clivage ou une mise à l'écart momentanée de certains facteurs déterminant le processus de décrochage scolaire. Afin de prendre en considération les déterminants internes à l'école, et en particulier les modalités d'apprentissage et d'enseignement et les relations entre élèves et enseignants (Bautier \& Rochex, 1997 ; Blaya, 2010 ; Blaya, Gilles, Plunus, \& Tièche Christinat, 2011 ; Glasman \& CEuvrard, 2004), la première orientation met en œuvre des modalités d'enseignementapprentissage proches de celles pratiquées dans l'enseignement spécialisé et suppose que l'école doit s'adapter à l'élève. Elle revient souvent à pratiquer ce que Henri-Panabière, Renard et Thin (2013) nomment une pédagogie du détour, qui pour contourner le blocage de l'élève consiste à « enseigner sans en avoir l'air et sans rien dire, en faisant faire et en mettant en activité sans interroger la nature des savoirs alors transmis» (p.74). Toutefois, l'introduction de changements didactiques peut s'avérer insuffisante et doit se doubler d'une réflexion sur ce que Martinez (2008) nomme les « irritants imputables à nos conceptions pédagogiques et aux programmes d'enseignement ». Autrement dit et tel que le soulignent d'autres travaux menés en sociologie et en sciences de l'éducation (Bressoux, 1994 ; Chevallard, 1985 ; CRESAS, 1981; Perrenoud, 1989), lutter contre le décrochage scolaire nécessite d'examiner les décisions prises dans la noosphère quant au choix des programmes et leur transposition au sein de la classe. De plus, modifier les contenus d'enseignement et approches didactiques tels que questionnés par l'équipe Eduscol ${ }^{1}$ risque de rester sans effet si « les méthodes et les contenus ne sont pas pensés dans la proximité du milieu social d'appartenance» (Pain, 2012, p. 199). La seconde orientation revient à privilégier une approche quasi thérapeutique, qui permet au jeune de se (re) construire comme sujet de sa vie et du monde (Tortolano, 2014), de panser les blessures narcissiques, d'augmenter la motivation et l'estime de soi ou encore de combler des lacunes spécifiques d'apprentissage afin de permettre à l'élève de renouer avec la réussite et de s'inscrire dans un projet de formation. Ainsi ce que Cellier (2011) nomme le "prendre soin pédagogique» ne peut être efficace que s'il "s'inscrit dans une appréhension globale de l'enfant et de sa famille » (p. 52) ou qu'il s'insère en complément à d'autres actions relevant des facteurs internes à l'école, tel le contexte de classe et de vie (Guigue, 2013b), ou encore dans les objectifs poursuivis par les politiques scolaires (Glasman \& CEuvrard, 2004 ; Perron \& Veillette, 2012). Ces derniers constats confirment la nécessité d'une approche systémique ou écologique (Bronfenbrenner, 1999), qui permet de considérer dans leur complémentarité la multiplicité des facteurs et de tenir compte de l'ensemble des acteurs. La diversité des lieux d'accrochage témoigne non seulement des facteurs pris en compte, mais également de la qualité des interactions entre les différents acteurs (Guigue, 2013b) et des liens entre les dimensions individuelles, familiales, collectives et sociales que ces lieux mettent en scène (Tortolano, 2014).

La mise en place des deux orientations mentionnées s'effectue sous certaines conditions. En effet, les travaux menés sur les programmes destinés à des populations dites à risque repèrent de nouvelles formes d'organisation qui diffèrent des pratiques ordinaires développées dans l'enseignement public. L'effectif des élèves regroupés dans ces programmes et le nombre d'acteurs professionnels qui gravitent autour d'un élève en situation de décrochage résultent en partie de la prise en compte de plusieurs déterminants du décrochage. Par ailleurs, leur diversité et leur complexité nécessitent le regard et l'intervention de plusieurs professionnels autour d'un élève. Ainsi ce qui faisait figure de particularité lors de la prise en charge d'élèves relevant de l'enseignement 
spécialisé, est généralisé à l'ensemble des élèves qui ne s'inscrivent pas dans une carrière scolaire normée. La majorité des dispositifs de lutte contre le décrochage adopte et opérationnalise le changement paradigmatique amorcé dans les années 1980 en avançant l'idée que les enseignants ne peuvent assumer à eux seuls l'accompagnement de tous les élèves. Dès lors une pluralité d'acteurs venus de champs professionnels variés caractérise les prises en charge et activités mises en place, entrainant ipso facto une modification du métier d'enseignant.

7 Par ailleurs si l'élève et sa singularité demeurent le centre de l'attention, les analyses de plusieurs dispositifs de prévention ou d'accrochage scolaire soulignent également la nécessaire collaboration avec la famille, et ce dès la mise en œuvre d'un projet d'accrochage (Houssemand \& Meyers, 2012; Poncelet, Pelt, Dierendonck, Mancuso, \& Kerger, 2013 ; Tièche Christinat, Angelucci, \& De Chambrier, 2016). Reconnus et légitimés dans les textes officiels comme des partenaires obligés de l'école, les parents jouent à double titre un rôle essentiel pour la réussite de l'accrochage. D'une part, les professionnels des dispositifs étudiés vont solliciter des alliances avec les parents pour étayer et consolider les actions éducatives et pédagogiques menées au sein du dispositif. D'autre part, ces professionnels vont mener diverses actions auprès des parents, afin de modifier le regard qu'ils portent sur l'école ou sur leur enfant.

Dans le cadre de cet article ${ }^{2}$, l'intérêt que nous portons au phénomène du décrochage s'inscrit dans une interrogation portant sur les déterminants intrinsèques au système scolaire et restreinte aux pratiques pédagogiques qui constituent une pièce importante du décrochage scolaire. Si les travaux précédemment cités décrivent plusieurs registres de fabrication du décrochage scolaire au sein de l'école publique et obligatoire, certains pointent en particulier l'opacité pour les élèves des modalités de travail et d'évaluation ainsi que l'effacement progressif des objectifs scolaires, à savoir l'appropriation de savoirs structurés en discipline. L'interprétation que les élèves en situation de décrochage ont du travail scolaire indique la présence de nombreux malentendus socio-cognitifs, qui contrarient voire empêchent l'accrochage à des activités d'apprentissage de savoirs académiques (Bautier 2003; Bautier \& Goigoux, 2004). Or on peut supposer que les activités menées pour favoriser la persévérance scolaire consistent entre autres à pallier les ratés pédagogiques et à créer, au sein de nouveaux dispositifs, comme le soulignent Lessard, Poirier et Fortin (2012), des alliances entre l'enseignant et l'élève.

\subsection{Le MATAS, un dispositif d'accrochage particulier}

Depuis 2006 et dans le cadre de la politique socio-éducative cantonale, les autorités scolaires du canton de Vaud (Suisse) ont ajouté à leur offre de prestations à l'égard des mineurs les Modules d'Activités Temporaires et Alternatifs à la Scolarité (MATAS). Mis en place pour des élèves en âge de scolarité obligatoire ${ }^{3}$ ( 4 à 16 ans) et considérés comme à risque de rupture scolaire, ces modules ont pour but de soutenir l'élève « dans sa capacité d'acquisition et dans sa capacité de motivation à l'apprentissage, dans les situations à haut risque de rupture scolaire ou de désinvestissement des apprentissages » (DFJC, 2006, Art. 5.2.5).

10 Ces dispositifs, assimilables à ce que Barrère (2013) nomme une " alternative ponctuelle au fonctionnement ordinaire de l'école» (p. 100), reposent sur le travail d'une dyade formée par un enseignant et un éducateur et visent la poursuite de la scolarité de l'élève à risque (RLEO, 2012). L'examen du travail mené conjointement par l'enseignant et 
l'éducateur engagés dans les MATAS indique un partage plutôt traditionnel des rôles et territoires professionnels, à savoir la gestion du travail scolaire pour l'enseignant et la gestion des comportements inadéquats ou difficiles ainsi que l'intervention auprès des familles des élèves pour l'éducateur (de Chambrier, Tièche Christinat, Chapuis, Angelucci, \& Liechti, 2014).

Outre le fait de prendre en charge l'élève orienté en MATAS, les deux professionnels engagés dans ces dispositifs ont une mission de médiation entre l'établissement scolaire, ses agents et la famille. Leurs rôles consistent à rapprocher parents et enseignants afin qu'ils puissent modifier le regard qu'ils se portent mutuellement. Dans le but de garantir au mieux l'exécution de ces missions délicates, les professionnels engagés par l'institution scolaire et socio-éducative doivent être au bénéfice d'un diplôme en enseignement ou en travail social et comptabiliser plusieurs années d'expérience professionnelle dans leur métier.

Durant les trois mois de prise en charge (renouvelable une fois), l'élève fréquente en alternance sa classe d'origine (à raison de deux à trois jours par semaine) et le MATAS situé hors de l'établissement scolaire. Ces particularités organisationnelles et structurelles qui font vivre à l'élève de multiples transitions - d'un lieu à l'autre, d'un professionnel à un autre et d'un groupe classe à l'autre - ont suscité à l'équipe lausannoise du LASALÉ ${ }^{4}$ nombre d'interrogations, dont celles portant sur la spécificité des contenus travaillés tout au long de la prise en charge et de leurs effets sur l'accrochage scolaire.

\section{Démarche}

13 Afin de caractériser et comprendre les activités développées pour enrayer le processus de désengagement d'élèves orientés temporairement dans un dispositif MATAS, notre démarche qualitative et compréhensive s'est appuyée sur la description par les professionnels des activités éducatives et pédagogiques qu'ils conduisent au sein des MATAS. Plus particulièrement, il s'agissait de questionner la dimension intrinsèque de cette activité en portant attention aux significations qu'ils donnent aux situations d'apprentissage et programmes mis hebdomadairement en œuvre auprès des élèves. Toutefois, et bien que le décrochage scolaire résulte du cumul de plusieurs facteurs de risque, nécessitant de fait une réponse multidimensionnelle, cette étude avait pour objectif de repérer le travail effectué qui se rapportait essentiellement aux facteurs intrinsèques au système scolaire. Au vu de l'importance et la diversité des facteurs pédagogiques et didactiques mentionnés ci-dessus, il apparaît nécessaire d'interroger aussi finement que possible la nature des leviers actionnés par les professionnels au sein d'un dispositif pour atteindre les objectifs d'accrochage et de persévérance scolaire. Ces différents éléments conduisent à interroger les données en trois temps. Si la première question porte sur le sens attribué par les professionnels aux situations d'apprentissage proposées aux élèves pris en charge, la seconde interpelle l'opérationnalisation de ces approches décrites. La troisième interrogation quant à elle cherche à comprendre en quoi certaines activités participent à l'accrochage scolaire. 


\subsection{Récolte des données}

14 Les données utilisées pour cet article sont issues de dix focus groupes menés dans le but de recueillir les expériences et opinions des professionnels travaillant dans les MATAS. Deux groupes de discussion composés chacun de sept à neuf participants ont ainsi été formés, rassemblant pour l'un les éducateurs et pour l'autre les enseignants des dispositifs MATAS participant à la recherche. Conduits par deux chercheurs, ces groupes de discussion ont été menés à cinq reprises avec chaque groupe et ont permis d'aborder différents thèmes, telles que la procédure d'admission et de fin de prise en charge, la collaboration entre professionnels MATAS, le travail avec les familles, ou encore la place des savoirs et le travail scolaire au sein du dispositif. Les analyses qualitatives présentées ici portent plus particulièrement sur le focus groupe ayant porté sur la place des savoirs et le travail scolaire et s'appuient également sur les données récoltées lors des autres focus groupes.

\subsection{Procédure d'analyse}

L'analyse des données réalisée s'inscrit dans le processus proposé par Miles et Huberman (2003). Suite à la transcription complète des enregistrements (durée moyenne des focus groupes de 90 minutes; durée totale $14 \mathrm{~h} 30$ ), un premier codage suivant les principes d'une analyse de contenu (Van Campenhoudt \& Quivy, 2011) a été effectué en dyade de chercheurs, faisant ainsi émerger les principales catégories d'analyse. Réalisées de manière itérative et soutenues par l'utilisation du logiciel HyperResearch $\odot$, les étapes de lecture, de codage et de discussion entre chercheurs au sujet des catégories émergentes comme de la segmentation en unités de sens - ont permis la stabilisation du système de catégories commune à l'ensemble des focus. Celle-ci a ensuite été systématiquement appliquée à l'ensemble des transcriptions. Les données non retenues après ce premier filtrage ont conduit à l'établissement de grilles spécifiques à certains focus et ont fait l'objet d'une seconde analyse.

\section{Approches et registres d'activité au service de l'accrochage}

Les analyses que nous avons pu développer grâce à une condensation des données faites en différentes étapes ont permis de distinguer plusieurs approches du travail effectué par les enseignants et/ou par les éducateurs et d'en saisir les orientations. Les tendances réparatrices de l'élève ou restauratrices de la pédagogie dont nous avons cité plus haut l'existence dans d'autres dispositifs d'accrochage, bien que présentes dans les propos tenus se déclinent légèrement différemment sous la forme de trois approches de nature psycho-clinique, socio-éducative ou encore académique. Un deuxième niveau d'analyse a permis de distinguer deux registres d'activités - le registre d'activités alternatives ou le registre d'activités scolaires -distincts à la fois par le lieu, le temps et le type d'activité. Si les propos relevés présentés ci-dessous permettent de comprendre les raisons qui président au choix des activités ainsi que le sens qui leur est attribué, ils illustrent aussi certains risques que nous déclinons dans la troisième partie de cet article et qui pourraient infléchir les objectifs visés. 


\subsection{Trois approches pour viser l'accrochage} professionnels interrogés afin de favoriser l'accrochage scolaire des élèves pris en charge au sein du dispositif MATAS. La première approche relève de la psychologie et tend à favoriser par une démarche de type clinique la " (re) construction» de l'élève en tant qu'individu. Se dessine chez les professionnels MATAS un consensus quant à la nécessité de travailler ce rapport de "Soi à Soi » qui s'exprime à travers la recherche du développement de l'estime de soi, de l'autonomie, de la confiance en soi, de la persévérance et de l'investissement face à la tâche. Ainsi, l'illustre le propos de cette enseignante, « (...) nous on prend le relai [ndlr : de l'enseignante de classe], on est là pour le [ndlr : l'élève] revaloriser, pour le remettre en confiance, travailler l'estime de soi, sur sa motivation" [ens_1]. Plus spécifiquement, les professionnels interrogés s'accordent à dire que le travail sur la motivation représente un aspect incontournable de leur prestation. La remotivation parait essentielle, en ce sens qu'elle est pensée d'une part comme appui pour permettre à l'élève de réinvestir ses apprentissages scolaires, mais aussi d'autre part comme levier pour pouvoir s'insérer socialement en tant qu'homme et citoyen "c'est la remotivation à l'apprentissage, mais au sens large, ça veut dire avoir envie de progresser, avoir envie d'apprendre, avoir envie de chercher, d'avoir envie de participer aux activités de sa ville, d'être euh un citoyen responsable, c'est tout ça pour moi remotiver à l'apprentissage (...) ) [ens_3].

18

visée réparatrice de l'élève avec une coloration cependant davantage liée à la socialisation de ce dernier. Ce travail de "Soi à l'Autre» prend principalement forme autour des aspects relationnels et comportementaux et de la prise en compte des codes et conventions qui régissent les interactions sociales. Ainsi les participants aux focus groupe signalent l'importance accordée au travail portant sur le comportement de l'élève vis-àvis de ses pairs et des adultes, sur le respect de l'autre de même que sur le respect des règles et règlements. Les compétences communicationnelles et collaboratives révélatrices de certains comportements semblent constituer un axe de travail partagé ainsi que l'expriment ces enseignants «(...) Je trouve que c'est au niveau des interactions entre les enfants que c'est très intéressant de travailler leur comportement, les coups de pied sous la table, les "je t'écoute pas" (...)» [ens_2], "(...) je leur enseigne les bases du comportement, enfin du comportement dans une classe, je me déplace d'un endroit à l'autre de façon normale, je ne me roule pas par terre, je peux patienter, etc. etc." [ens_3]. Le développement des habiletés sociales des élèves fait également consensus chez les professionnels interrogés qui visent ainsi des apprentissages permettant à l'élève de se comporter de manière appropriée non seulement à l'école, mais aussi dans d'autres contextes.

L'acquisition de connaissances disciplinaires, en français et mathématiques et domaine des sciences humaines et sociales plus accessoirement, fonde la troisième approche. Là encore, les professionnels expriment un degré d'accord très élevé sur la nécessité de travailler des connaissances de nature académique (relation de "Soi aux Connaissances ») à la différence près que certaines divergences apparaissent dans les formes, les pratiques et les orientations pédagogiques adoptées. Ainsi, les avis des professionnels au sujet du «maintien du niveau scolaire de l'élève» tendent à être consensuels malgré des variations qui semblent dépendre davantage des structures au sein desquels les professionnels œuvrent que de leur sphère professionnelle. S'il s'agit en effet d'un 
objectif prioritaire pour certaines structures, d'autres « (...) postule[nt] qu'il y a des priorités qui sont plus importantes que simplement le maintien du niveau de l'élève " [édu_1]. Tous les participants s'entendent cependant sur le fait que le MATAS n'a pas pour but de rattraper un retard scolaire.

De ces trois approches dédiées à l'accrochage scolaire, il faut relever le poids important accordé aux approches de nature psycho-clinique et socio-éducative laissant de fait apparaitre une relative focalisation des professionnels MATAS sur des approches liées à la personne, à sa reconstruction et à sa socialisation. Il convient en outre de noter que si les discours analysés comportent des traces au sujet du travail sur les connaissances disciplinaires, il semblerait que les enjeux cognitifs et didactiques demeurent toutefois à l'arrière-plan des approches visant à réparer l'individu. Ainsi, bien que l'orientation quasi thérapeutique visant à la $(\mathrm{re})$ construction de l'élève et l'orientation ambitionnant sa restauration pédagogique ne semblent totalement indépendantes, il demeurerait toutefois la représentation selon laquelle la (re) construction de l'élève et sa socialisation seraient des étapes antérieures à celles des apprentissages (Barataud, 2003), comme si le registre des apprentissages ne saurait être producteur d'effets de socialisation et de reconstruction (Terrail, 2006).

\subsection{Des registres alternatifs et scolaires}

Utilisés par les professionnels afin d'opérationnaliser les trois approches susmentionnées, deux registres d'activités, cloisonnés dans le temps et l'espace, apparaissent à la lecture $\mathrm{du}$ corpus. Proposées en alternance durant le programme quotidien, la première forme consiste en des activités alternatives qui se distinguent facilement de la seconde nommée moments scolaires (figure 1).

Figure 1 : exemple de planification hebdomadaire extraite d'un concept pédagogique MATAS I

Image 2000021D000040170000093A52699B40.wmf

22 Lors des activités alternatives, il s'agit de "faire de l'éducatif» [édu_2] en utilisant des supports qui diffèrent de ceux utilisés régulièrement dans le cadre de la scolarité publique et obligatoire. Modelage, land art, visite à la ferme, atelier bois, course en montagne, escalade, sortie en bateau sur le lac, cuisine, mais aussi réalisation d'un journal informatisé ou d'une émission de radio représentent quelques-unes de ces activités auxquelles deux-tiers du temps de présence des élèves au MATAS sont en moyenne consacrés. Si la conduite du groupe d'élèves s'effectue la plupart du temps en présence des deux professionnels MATAS (de Chambrier et al. 2014), certaines activités alternatives requièrent en sus la présence d'intervenants externes experts de leur domaine pouvant également être directement liés au domaine du soin (fermier, potier, art-thérapeute ou l'équi-thérapeute, par exemple).

Les moments scolaires aussi dénommés par plusieurs participants «moments purement scolaires » sont pour leur part généralement décrits comme un tableau illustrant la classe de manière prototypique, mettant en avant des éléments fortement connotés : une enseignante, un espace-classe, un tableau noir, des fiches et des cahiers pour réaliser des tâches en mathématiques ou en français principalement. Ainsi l'illustrent les propos de ces enseignants «(...) moi j'aime beaucoup définir le travail scolaire comme vient de le dire ens_ $x$ euh des fiches, posé à une table comme à l'école, c'est ça que j'appelle du travail purement 
scolaire » [ens_4] ou encore, "(...) le purement scolaire c'est des fiches de maths, voilà, ils font des additions, soustractions, ou bien des verbes, des choses comme ça. Pour moi ça c'est vraiment le purement scolaire.» [ens_5]. Ainsi le travail purement scolaire n'interpelle pas explicitement la place de savoirs académiques précis ou les plans d'études, mais il affiche surtout la présentation très traditionnelle de ceux-ci, et indirectement la posture que l'enseignant occupe dans la classe. Si un consensus se forme parmi les professionnels interrogés sur le principe d'alternance entre "activités alternatives» et "moments scolaires " ainsi que sur le temps alloué à chacune de ces formes, certains propos indiquent toutefois un questionnement des pratiques voire la mise en place de pratiques diversifiées montrant ainsi une non-adhésion à la reproduction de la forme scolaire traditionnelle. Pour l'une des équipes par exemple, il s'agit de s'interroger sur « la bonne manière de faire du scolaire » : "(...) il a y six ans quand on a ouvert le MATAS, on se mettait en rond (...) on se demandait comment ça allait, vraiment c'était très éducatif, purement éducatif, et puis petit à petit on s'est rendu compte que c'était désécurisant (...). Maintenant, je trouve que le fait d'avoir réintroduit un peu de l'école pure, avec une maitresse, un tableau noir, on lève la main, c'est les mêmes règles, on insiste beaucoup là-dessus, ça amène une continuité avec l'école (...)" [édu_4] alors qu'une autre équipe a pris la décision de complètement évacuer les «moments scolaires» de leur grille horaire tablant sur les bénéfices d'une rupture davantage marquée avec le cadre scolaire prototypique. Ainsi le fait remarquer cette enseignante "(...) je ne fais absolument pas de scolaire avec les élèves donc c'est une parenthèse qu'on met, c'est des choses qu'on met au clair avec les parents lors du bilan de début de prise en charge, (...) on ne fait pas de fiches de maths ni de français, le programme scolaire il est mis de côté (...).» [ens_3]. Ces divers propos indiquent que la coexistence des deux registres, bien qu'agréée et souhaitée par les politiques, n'est pas toujours effective, mais aussi que le ratio de chaque registre s'effectue suivant des processus dynamiques propres à chaque MATAS.

\subsection{Le sens des activités alternatives}

L'analyse des données met en évidence que les activités alternatives seraient autant d'occasions d'opérationnaliser des approches de nature psychologique et socio-éducative que d'aborder dans certains cas du moins, des contenus disciplinaires (approche de nature académique). Les moments scolaires resteraient pour leur part davantage confinés à une approche de nature académique. Bien que ces résultats méritent d'être étayés par des observations, ce constat exprime de manière implicite et non partagée par tous les participants, la conception d'une certaine continuité entre les registres alternatifs et scolaires, le premier impliquant des aspects éducatifs et le second faisant appel à des connaissances disciplinaires. Si certaines activités alternatives semblent davantage pensées et basées sur des approches de nature psychologique et socio-éducative, d'autres intègrent donc plus explicitement une approche de nature académique. À titre d'illustration, alors que l'escalade ou l'approche par le cheval mises en œuvre par les professionnels interrogés ont pour but d'accroître la confiance en soi, l'estime de soi, la gestion des émotions ou encore le lien et la confiance en l'autre, d'autres activités comme la radio et la cuisine sont pensées afin de développer également une approche de nature académique. En ce sens, alors que l'activité cuisine implantée dans l'ensemble des dispositifs étudiés relève certes largement des approches de nature psychologique et socio-éducative "s'ils sont trois [ndrl: durant l'activité cuisine] il y a l'organisation, le partage des tâches, le partage de l'espace, comment on se parle. La cuisine n'est pas très grande, c'est une 
cuisine d'appartement donc il faut aussi toutes ces compétences de groupe, des compétences sociales." [ens_6], un important discours met également en évidence une approche académique "Oui, il y a les mesures aussi. Tu dois peser, on va faire les commissions avec, donc il faut quand même qu'ils sachent lire la liste des commissions (...).» [ens_7], "les changements d'unités aussi, les litres, décilitres. (...). On mobilise des connaissances scolaires.»[ens_4]. L'intégration d'une approche basée sur les connaissances disciplinaires se retrouve en outre dans le cadre de l'activité radio, celle-ci s'appuyant par ailleurs aussi sur des approches psychologiques et socio-éducatives. L'actualisation de l'approche académique (sensibilisation de l'élève au langage écrit et oral) est ici réalisée par la rédaction d'un texte portant sur un sujet libre et sa lecture à des fins d'enregistrement. Le produit final de l'activité consiste en la réalisation et le dépôt d'un podcast sur une radio en ligne ainsi que sa diffusion dans la classe d'origine de l'élève ou sa famille. Ces deux activités ont en commun le fait de contribuer à l'élaboration d'une continuité entre le MATAS et la classe d'origine ou le MATAS et la famille. En effet, le produit réalisé par l'élève (un podcast, un gâteau,...) et son partage dans d'autres lieux permet de créer du lien entre le MATAS et d'autres acteurs évoluant autour de l'élève. Ce produit agirait selon les participants comme objet médian, permettant d'échanger autour du travail réalisé avec d'autres acteurs entourant l'élève (famille, enseignants, pairs) et in fine de faire évoluer le regard porté sur ce dernier. Ces deux activités se distinguent toutefois dans l'espace-temps qui leur est consacré. Alors que dans le cadre de l'activité Cuisine les connaissances disciplinaires sont travaillées spécifiquement durant le temps dédié à l'activité alternative, la rédaction du texte servant à l'émission Radio déborde de cet espace-temps, puisqu'il mobilise l'enseignant également lors du moment scolaire. Ainsi le cloisonnement des activités n'est plus effectif dans ce dernier cas, mais toutes deux (alternatives et scolaires) participent à l'illustration de savoirs académiques utiles et utilisables. À ce stade, il convient en outre de formuler l'hypothèse selon laquelle le professionnel aux commandes de l'activité (l'éducateur ou l'enseignant) joue un rôle important dans les approches mobilisées, la posture et le regard portés sur l'activité orientant possiblement les approches mises en évidence.

Au-delà de l'actualisation des trois approches décrites ci-avant, les activités alternatives favorisant très souvent la réalisation de projets concrets semblent être particulièrement exploitées comme levier motivationnel «la motivation il peut la retrouver dans les activités alternatives, dans le contact qu'on établit avec lui, et puis ces gamins, il y a rien à faire, ils sont quand même attachés au résultat, que ce soit les tout grands qui se font vider de l'école, ou les plus petits de $5^{e}$. Le résultat c'est quand même important pour eux!»[ens_6]. D'autres propos renforcent l'importance accordée à la motivation comme condition et non comme la résultante d'apprentissage de savoirs académiques, «c'est antinomique de pouvoir maintenir le niveau tout en faisant encore ce qu'ils font encore à l'école. Enfin moi, je passerais beaucoup plus par carrément tout casser, pas tout casser, mais casser le rythme de ce qui était fait à l'école pour pouvoir les motiver en espérant que cette petite bulle d'air pourra leur donner un élan pour raccrocher » [ens_2].

Le registre alternatif sur lequel est fondé le concept de MATAS postule « qu'il est plus aisé pour l'enfant de travailler ses difficultés, de créer des expériences positives et de retrouver une certaine confiance en lui même au travers des activités alternatives $»^{5}$ s'inscrit de fait particulièrement bien dans ce que Henri-Panabière et ses collègues (2013) nomment la pédagogie du détour. Mis en évidence dans le cadre d'une étude portant sur les Ateliers Relais comme : 
(...) une tactique de l'esquive ou du contournement, sorte de feinte pédagogique pour ne pas placer frontalement les collégiens face aux savoirs et aux formes d'apprentissage auxquels leurs parcours (y compris scolaires) les ont rendus réfractaires, et pour "réconcilier» progressivement les collégiens avec la scolarisation (p. 4)

l'usage du registre alternatif vise effectivement à atteindre l'élève de manière indirecte afin de favoriser son engagement dans la tâche, sa persévérance et de réaliser in fine de nouveaux apprentissages. Cette orientation pédagogique ne demeure toutefois pas sans poser de questions sur les risques potentiels d'engendrer ou d'accentuer des malentendus socio-cognitifs et interroge plus avant les notions de transfert de compétences. En effet, le recours à des activités alternatives qui renforce à la logique du réconfort évoquée par Bautier (2003) afin de motiver à l'apprentissage en rendant une tâche plus attractive peut en masquer l'enjeu cognitif entravant les apprentissages et ne permettant que difficilement de redonner un sens aux activités scolaires pratiquées en classe.

\section{Discussion}

Les approches psycho-clinique, socio-éducative et académique, qui caractérisent le travail au sein du dispositif MATAS ont certes pour cible l'élève en situation de décrochage. Toutefois comme le mentionnent plusieurs travaux scientifiques réunis dans le livre de Gilles, Potvin et Tièche Christinat (2012), elles ne peuvent porter des fruits que si d'autres actions destinées à l'entourage de l'élève sont menées. La création de «relations entre ceux qui agissent dans l'école et ceux qui œuvrent à l'extérieur» (Balas, 2013, p. 3) apparaît nécessaire pour assurer non seulement la réussite de la prise en charge, mais aussi la continuité éducative d'un lieu à l'autre, d'une personne à l'autre. En effet, la création ou le maintien d'alliances éducatives entre les différents acteurs appartenant à la sphère scolaire (enseignant-e-s de classe d'origine, directeurs d'établissement), familiale (père, mère, fratrie,...), thérapeutique (logopédistes, psychologues, médecins,...) ou juridique (service de protection de la jeunesse, juge des mineurs) semblent conditionner l'accrochage de l'élève. L'absence de mobilisation des différents acteurs et plus particulièrement l'absence d'une réflexion sur les apports et les rôles de chacun conduirait selon Brüggen (2010) à long terme à un échec du dispositif. Les données que nous avons extraites pour analyser le travail d'accrochage effectué par les professionnels pourraient donner une image bien incomplète, voire erronée du travail effectué. En effet, à côté de la prise en charge de l'élève dans sa singularité et que nous avons analysé cidessus, l'étude plus large du dispositif MATAS montre l'importance de la collaboration entre les différents acteurs qui gravitent autour de l'élève (de Chambrier et al., 2014). Par ailleurs, le paradigme fondateur de l'école vaudoise actuelle reconnaissant la nécessaire collaboration avec les parents (Loi sur l'Enseignement Obligatoire) et les services pédagothérapeutique, influence fortement l'orientation du travail chez les professionnels du MATAS. De façon similaire aux constats de Millet et Thin (2014) et aux pistes pédagogiques proposées par Goémé, Hugon et Taburet (2012), enseignants et éducateurs du MATAS contribuent à ( $r$ )établir des relations saines entre l'élève à risque et les adultes, notamment avec le ou les enseignants de sa classe d'origine. Il en va de même pour le travail avec les familles des élèves pris en charge dont l'un des buts pour les professionnels est d'œuvrer dans une optique "pacificatrice» afin de revaloriser et réhabiliter les élèves aux yeux de leurs parents et de leurs enseignants ordinaires. Le changement de regard sur l'élève ou l'enfant qui constitue un des objectifs déclarés par 
les professionnels sert aussi et de manière plus indirecte à modifier les relations entre les parents et l'institution scolaire.

Plus avant, l'étude de ces dispositifs interroge sur la psychologisation des difficultés d'apprentissage et les différentes réponses proposées. Nous retrouvons dans le discours des professionnels interrogés comme dans les textes officiels, une focalisation ou un accent marqué sur les enjeux psychoaffectifs plutôt que sur les enjeux cognitifs et didactiques. Le travail pédagogique et les apprentissages scolaires se situent en seconde zone d'intérêt et ne sont pas priorisés, puisque considérée sans urgence aucune, l'évaluation des élèves pouvant être suspendue durant le MATAS. De plus, la prise en charge ponctuelle des élèves par exemple, par une art-thérapeute ou autre équithérapeute questionne également sur les pratiques de motivation qui à notre sens comportent les risques de s'inscrire dans un processus plus large de délégation du traitement des difficultés scolaires auprès d'autres spécialistes issus du champ médical. L'importance du recours à la motivation reflèterait aussi cette tendance à prendre en compte la dimension psychologique et à omettre la dimension scolaire. La tension entre une conception de l'enseignement qui oppose le plaisir à l'apprentissage et qui soutient que l'épanouissement de l'élève est une condition ou un prérequis à tout apprentissage a en effet été apparente durant les focus groupes. Si ces représentations peuvent conduire certains acteurs à s'affranchir totalement de la forme scolaire, leur remise en question permet à d'autres professionnels d'interroger le fait d'avoir « aussi peu de scolaire ».

Pour terminer, les registres alternatifs et scolaires cités par les professionnels comme les deux piliers des activités proposées au MATAS peuvent se légitimer de différentes manières. D'une part, le choix de ces deux registres peut être directement imputé à l'identification de l'exercice professionnel de chacun, soit à l'enseignement et à la prise en charge socio-éducative. Leur existence semble ainsi donner sens à l'organisation structurelle du MATAS et accrédite le prescrit institutionnel qui impose la co-présence d'un enseignant et d'un éducateur social. Ils permettent également aux dires de certains professionnels d'établir l'originalité de la prise en charge par rapport aux autres prestations pédagogiques ou pédago-thérapeutiques offertes par l'école, voire de justifier l'orientation MATAS aux parents qui s'interrogent sur une prise en charge extérieure à l'école de leur enfant tout en soulignant, par la présence d'un registre d'activités scolaires, le maintien d'une identité scolaire. De plus, l'existence de deux registres favoriserait également une meilleure identification des rôles de chaque professionnel par l'élève. D'autre part, leur choix et le poids inégal accordé à chaque registre semblent se justifier également par le repérage de certains facteurs propres au décrochage scolaire en particulier par une surreprésentation des facteurs comportementaux, et ce, au détriment d'autres facteurs. Cependant, bien qu'accueillant des élèves qualifiés de perturbateurs et ce faisant facilitant le travail de l'enseignant, la prise en charge au moyen d'activités qui éloignent le registre scolaire en privilégiant l'action immédiate et la manipulation, pourrait par ailleurs s'avérer déloyale vis-à-vis de l'école et des enseignants et même contreproductive (Barrère, 2015). 


\section{BIBLIOGRAPHIE}

Baeriswyl, D., Savoy, B., \& Tièche Christinat, C. (2013). Viser l'accrochage : pratiques pédagogiques et alliances dans des structures destinées à des élèves ou des jeunes en difficultés. Education \& Formation, $n^{\circ} e-300,15-26$.

Balas, G. (2012). Lutter contre le décrochage scolaire. Vers une nouvelle action publique régionale. Paris : Fondation Jean Jaurès.

Barataud, D. (2003). Interoger les objets de savoir eux-mêmes, une piste de réflexion. La nouvelle revue de l'AIS, 24(203), 95-214.

Barrère, A. (2013). Les établissements scolaires à l'heure des "dispositifs". Carrefours de l'éducation, 36, 9-13.

Bautier, E. (2003). Décrochage scolaire. Genèse et logique des parcours : Prévenir les ruptures scolaires. VEI Enjeux, 132, 30-45.

Bautier, E., \& Goigoux, R. (2004). Difficultés d'apprentissage, processus de secondarisation et pratique enseignantes : une hypothèse relationnelle. Revue française de pédagogie, 148, 89-100.

Bautier, E., \& Rochex, Y. (1997). Apprendre des malentendus qui font la différence. In J.-P. Terrail (Ed.), La scolarisation de la France. Critique de l'état des lieux (pp. 105-122). Paris : La dispute.

Bernard, P.-Y. (2011). Le décrochage scolaire. Paris : PUF (QSJ).

Blaya, C. (2010). Décrochages scolaires. L'école en difficulté. Bruxelles : De Boeck.

Blaya, C. (2013). Le décrochage scolaire dans les pays de l'OCDE. Regards croisés sur l'économie (2), 69-80.

Blaya, C., Gilles, J. L., Plunus, G., \& Tièche Christinat, C. (2011). Accrochage scolaire et alliances éducatives : vers une intégration des approches scolaires et communautaires. Education et francophonie, XXXIX : 2, 227-249.

Bonnéry, Stéphane. (2007). Comprendre l'échec scolaire. Élèves en difficultés et dispositifs pédagogiques. Paris : La Dispute.

Bressoux, Pascal. (1994). Note de synthèse [Les recherches sur les effets-écoles et les effetsmaîtres]. Revue française de pédagogie, 108(1), 91-137.

Broccolichi, S., \& Ben-Ayed, C. (1999). L'institution scolaire et la réussite de tous aujourd'hui :" pourrait mieux faire". Revue française de pédagogie, 129(1), 39-51.

Bronfenbrenner, U. (1999). Environments in developmental perspective: theoretical and operational models. In S. L. Friedman \& T. D. Wachs (Eds.), Measuring environments across the life span: Emergin mathods and concepts. Washington, D.C. : American Psychological Association Press. Brüggen, S. (2010). School disaffection and adolescent identity. The International Journal on School Disaffection, 7(2), 40-44.

Cellier, H. (2011). Effets et limites de l'action des "référents dédiés" en Programme de réussite éducative. La nouvelle revue de l'adaptation et de la scolarisation, 56(4), 45-52. 
Chevallard, Yves (Ed.). (1985). La transposition didactique du savoir savant au savoir enseigné. Grenoble : Ed. La Pensée Sauvage.

Colombo, M. (2010). Dispersione scolastica e politiche per il successo formativo. Trento : Erickson.

CRESAS. (1981). L'échec scolaire n'est pas une fatalité. ESF.

de Chambrier, A.-F., Tièche Christinat, C., Chapuis, J., Angelucci, V., \& Liechti, L. (2014). La collaboration entre enseignants et éducateurs travaillant dans une structure au service de l'accrochage scolaire. Paper presented at the Décrocher n'est pas une fatalité. Le rôle de l'école dans l'accrochage scolaire. Luxembourg, 14-15-16 mai 2014.

Dubet, F. (2005). Les accidentés de l'école. In G. Brandibas \& R. Fourastié (Eds.), Les accidentés de l'école (pp. 23-30). Paris : L'Harmattan.

Dubet, F. (2014). Préface. In T. Berthet \& J. Zaffran (Eds.), Le décrochage scolaire. Enjeux, acteurs et politiques de lutte contre la déscolarisation. (pp. 9-17). Rennes : Presses Universitaires de Rennes.

Flavier, E., \& Moussay, S. (2014). Répondre au décrochage scolaire. Expériences de terrain. Bruxelles : De Boeck.

Fortin, L., Marcotte, D., Potvin, P., Royer, É, \& Joly, J. (2006). Typology of students at risk of Dropping out of school: Description by personal, family and school factors. European Journal of Psychology of Education, 21(4), 363-383.

Fortin, L., Marcotte, D., Royer, É., \& Potvin, P. (2005a). Facteurs personnels, scolaires et familiaux différenciant les garçons en problèmes de comportement du secondaire qui ont décroché ou non de l'école. Nouveaux cahiers de la recherche en éducation, 8(2), 79-88.

Fortin, L., Marcotte, D., Royer, É., \& Potvin, P. (2005b). Hétérogénéité des élèves à risque de décrochage scolaire : facteurs personnels, familiaux et scolaires. La réussite scolaire : comprendre et mieux intervenir. Québec: CRIRES et Les Presses de l'Université Laval.

Gilles, J.-L., Potvin, P., \& Tièche Christinat, C. (2012). Les alliances éducatives pour lutter contre le décrochage scolaire. Berne : Peter Lang.

Gilles, J.-L., Tièche Christinat, C., \& Delévaux, O. (2012). Origines, fondements et perspectives offertes par les alliances éducatives dans la lutte contre le décrochage scolaire. In J.-L. Gilles, P. Potvin \& C. Tièche Christinat (Eds.), Les alliances éducatives pour lutter contre le décrochage scolaire (pp. 3-18). Berne Peter Lang.

Glasman, D., \& CEuvrard, F. (2004). La déscolarisation. Paris : La Dispute.

Goémé, P., Hugon, M., \& Taburet, P. (2012). Le décrochage scolaire, des pistes pédagogiques pour agir. Lyon : Repères pour agir/Cahiers pédagogiques.

Guigue, M. (2013a). L'émergence d'expériences locales, enjeux pour les insitutions et pour les acteurs. In G. Boudesseul (Ed.), Du décrochage à la réussite scolaire : Expériences françaises et internationales. (pp. 39-52). Paris : L'Harmattan.

Guigue, M. (2013b). Les déchirements des institutions éducatives : jeux d'acteurs face au décrochage scolaire. Paris : L'Harmattan.

Henri-Panabière, G., Renard, F., \& Thin, D. (2013). Des détours pour un retour ? Pratiques pédagogiques et socialisatrices en ateliers relais. Revue française de pédagogie, 183, 71-82.

Houssemand, C., \& Meyers, R. (2012). Combattre le décrochage au Luxembourg à l'interface de l'école, de la famille et du marché de l'emploi : l'Action Locale pour Jeunes. In J.-L. Gilles, C. Tièche Christinat \& P. Potvin (Eds.), Alliances éducatives et décrochage scolaire. Berne : Peter Lang. 
Hugon, M.-A., \& Toubert-Duffort, D. (2011). Adolescence et décrochage : prévenir et répondre. Présentation du dossier. La Nouvelle revue de l'adaptation et de la scolarisation, 56(4), 7-12.

Lessard, A., Poirier, M., \& Fortin, M.P. (2012). La gestion de classe : Une alliance entre l'enseignant et l'élève. In J.-L. Gilles, P. Potvin \& C. Tièche Christinat (Eds.), Les alliances éducatives pour lutter contre le décrochage scolaire (pp. 83-108). Berne : Peter Lang.

Martinez, J.-P. (2008). La prévention de l'échec scolaire. Mythe ou réalité. Recherches enseignement, éducation et formation en espaces francophones, 7, 7-13.

Miles, M. B., \& Huberman, A. M. (2003). Analyse des données qualitatives : Méthodes en sciences humaines. Bruxelles : De Boeck.

Millet, M., \& Thin, D. (2005). Ruptures scolaires : l'école à l'épreuve de la question sociale. Paris : PUF.

Pain, J. (2012). Déscolariser le décrochage. In J.-L. Gilles, P. Potvin, \& C. Tièche Christinat (Eds.), Les alliances éducatives pour lutter contre le décrochage scolaire (pp. 193-205). Berne : Peter Lang.

Perrenoud, P. (1989). La triple fabrication de l'échec scolaire. Psychologie Française, 34(4), 237-245.

Perron, M., \& Veillette, S. (2012). Territorialité, mobilisation des communautés et persévérance scolaire : la diffusion d'une innovation sociale au Québec. In J.-L. Gilles, P. Potvin, \& C. Tièche Christinat (Eds.), Alliances éducatives et décrochage scolaire (pp. 169-189). Berne : Peter Lang. Poncelet, D., Pelt, Véronique, Dierendonck, Christophe, Mancuso, Giovanna, \& Kerger, Sylvie. (2013). « Partners », une recherche-action au Grand-Duché du Luxembourg visant la mise en place d'actions en direction des familles en vue d'instaurer une collaboration école-famille efficace et constructive favorable à l'accrochage scolaire des élèves : bilan de l'impact final dans une classe-cible. Revue Education et formation, $n^{\circ}$ e-302, 141-166.

Potvin, P., Fortin, L., \& Lessard, A. (2005). Le décrochage scolaire. In L. Massé, N. Desbiens \& C. Lanaris (Eds.), Troubles du comportement à l'école : prévention, évaluation et intervention (pp. 67-78). Montréal : Gaëtan Morin.

RLEO (2012). Règlement d'application de la loi du 7 juin 2011 sur l'enseignement obligatoire. Vaud, Suisse : DFJC.

Terrail, J.-P. (2006). La psychologisation de la difficulté intellectuelle. Un obstacle à la démocratisation scolaire. Le Journal des Psychologues, 236(3), 68-7.

Tièche Christinat, C., Angelucci, V., \& de Chambrier, A.-F. (2016). La famille, un allié essentiel à l'accrochage de l'élève. Education \& Formation, e-304, 25-37.

Tièche Christinat, C., Baeriswyl, D., Delévaux, O., Savoy, B., \& Cassagne, J.-M. (2012). De l'identification des facteurs de décrochage par des enseignants aux réponses pédagogiques et structurelles. In J.-L. Gilles, P. Potvin \& C. Tièche. Christinat (Eds.), Les alliances éducatives pour lutter contre le décrochage scolaire. (pp. 109-128). Berne : Peter Lang.

Tièche Christinat, C., \& Gilles, J.-L. (2013). Les alliances éducatives et accrochage scolaire. Education et formation, $n^{\circ} e-300$.

Tortolano, S. (2014). Décrochage scolaire : une polysémie à remettre en jeu. In A. Henrion \& J. Grégoire (Eds.), Adolescents et difficultés scolaires. Approche de la complexité (pp. 133-155). Bruxelles : Mardaga.

Van Campenhoudt, L., \& Quivy, R. (2011). Manuel de recherche en sciences sociales (4 éd.). Paris : Dunod. 


\section{NOTES}

1. Nous nous référons ici en particulier aux textes de Rochex (1995); de Bautier (2003) et de Bautier et Goigoux (2004).

2. La recherche présentée ici a été menée grâce au soutien du FNS (Fonds National Suisse de la Recherche Scientifique) et à l'octroi de la subvention nº 13 DPD3_136975.

3. L'école publique obligatoire débute dès l'âge de 4 ans et s'étend en règle générale sur 11 années. Les MATAS 1 accueillent des élèves âgés de 4 à 11 ans et qui fréquentent le cycle primaire. Les élèves du cycle secondaire, âgés de 12 à 15 ans, sont orientés vers les MATAS 2 .

4. Laboratoire International Accrochage scolaire et alliances éducatives, rattaché à la Haute Ecole Pédagogique du canton de Vaud, Lausanne.

5. Propos inscrits dans une flyer de présentation d'un des MATAS étudié.

\section{RÉSUMÉS}

Considérant l'importance des facteurs pédagogiques dans le processus de décrochage, cette recherche vise, au moyen de focus groupes $(n=10)$, la compréhension de l'activité des professionnels (éducateurs et enseignants) œuvrant au sein d'un dispositif d'accrochage (canton de Vaud, Suisse). Elle interroge la nature des leviers pédagogiques que les professionnels actionnent pour atteindre les objectifs d'accrochage et porte attention aux significations qu'ils donnent aux situations d'apprentissage mis en œuvre. L'analyse de contenu menée a permis de dégager trois approches privilégiées à des fins d'accrochage qui s'opérationnalisent par des registres d'activités alternatives et scolaires. Le poids important accordé aux approches de nature psycho-clinique et socio-éducative laisse apparaître une relative focalisation sur des situations relatives à la (re)construction de la personne et à sa socialisation. La place accordée à la motivation comme condition et non comme la résultante d'apprentissage des savoirs académiques relègue l'importance des enjeux didactiques et cognitifs (approche académique).

By means of focus groups $(n=10)$ and considering the importance of pedagogical components in the process of school dropout, this research aims to understand the activity of professionals involved in a program promoting school engagement on alterning courses (Canton of Vaud, Switzerland). The study examines the nature of pedagogical levers used by the professionals in order to achieve the objective of school engagement. It also focuses on meanings professionals give to the learning situations implemented. Content analysis conducted has yielded three approaches privileged to enhance school engagement, which are operationalized through academic or alternative activities. The substantial weight given to psycho-clinical and socioeducative approaches shows a relative focus on (re)construction of the pupil and his or her socialisation. The emphasis on motivation as a condition rather than the result of learning and academic knowledge relegates the importance of educational and cognitive issues. 
INDEX

Mots-clés : dispositif, accrochage scolaire, focus groupe, activités socio-éducatives, motivation

Keywords : program, school engagement, focus group, socio-educative activities, motivation

\section{AUTEURS}

CHANTAL TIÈCHE CHRISTINAT

VALÉRIE ANGELUCCI

LÉONIE LIECHTI 